DR. JIMENA RINALDI (Orcid ID : 0000-0003-4088-7067)

Article type : Research Article

\title{
A Spectroscopy-based Methodology for Rapid Screening and Characterization of Phytochrome Photochemistry in Search of Pfr-favoured Variants
}

Giuliano Tomás Anteloํ․ Maximiliano Sánchez-Lamas ${ }^{1}$, Fernando Alberto Goldbaum ${ }^{1}$, Lisandro Horacio Otero $^{1,2}$, Hernán Ruy Bonomi ${ }^{*}$, Jimena Rinaldi ${ }^{*}$

${ }^{1}$ Fundación Instituto Leloir, IIBBA-CONICET, Av. Patricias Argentinas 435, (C1405BWE) Ciudad Autónoma de Buenos Aires, Argentina.

${ }^{2}$ Plataforma Argentina de Biología Estructural y Metabolómica PLABEM, Av. Patricias Argentinas 435 (C1405BWE), Buenos Aires, Argentina.

*Corresponding authors email: jrinaldi@leloir.org.ar and hbonomi@leloir.org.ar

Keywords

Screening methodology; bacteriophytochrome; BphP; biliverdin IX $\alpha$; photochemistry, photocycle

This article has been accepted for publication and undergone full peer review but has not been through the copyediting, typesetting, pagination and proofreading process, which may lead to differences between this version and the Version of Record. Please cite this article as doi: $\underline{10.1111 / \text { PHP.13313 }}$

This article is protected by copyright. All rights reserved 


\section{ABSTRACT}

Phytochromes are photosensitive proteins with a covalently bound open-chain chromophore that can switch between two principal states: red-light absorbing Pr and far-red light absorbing Pfr. Our group has previously shown that the bacteriophytochrome from Xanthomonas campestris pv. campestris (XccBphP) is a bathy-like phytochrome that uses biliverdin IX $\alpha$ as a co-factor and is involved in bacterial virulence. To date, the XccBphP crystal structure could only be solved in the Pr state, while the structure of its Pfr state remains elusive. The aims of this work were to develop an efficient screening methodology for the rapid characterization and to identify $X c c \mathrm{BphP}$ variants that favour the $\mathrm{Pfr}$ form. The screening approach developed here consists in analyzing the UV-Vis absorption behaviour of clarified crude extracts containing recombinant phytochromes. This strategy has allowed us to quickly explore over a hundred $X c c B p h P$ variants, characterize multiple variants and identify Pfr-favoured candidates. The high-quality data obtained enabled not only a qualitative, but also a quantitative characterization of their photochemistry. This method could be easily adapted to other phytochromes or other photoreceptor families. 


\section{INTRODUCTION}

Phytochromes are photosensitive proteins with a covalently bound open-chain tetrapyrrole chromophore that can switch between two principal states: red-light absorbing (Pr) and far-red light absorbing (Pfr) (14). Typically, phytochromes present an N-terminal photosensory module composed of the PAS2-GAFPHY triad domain and an output module located towards the C-terminus. The output module can present a variety of functions which may include multiple or single enzymatic or non-enzymatic domains $(3,5,6)$. Phytochromes can be classified as "canonical" or "bathy" depending on whether they decay in the dark (dark conversion) towards a Pr or Pfr thermally stable state, respectively (2, 7-9). Since the pioneering works using purified phytochromes from natural plant sources (10-12), recombinant phytochromes have paved the way to more rapid biochemical, photochemical and structural studies (13-16).

In the last few years, our group has studied the structure, photochemistry and biological function of the bacteriophytochrome from the phytopathogen Xanthomonas campestris pv. campestris (XccBphP) (17-19). $X c c B p h P$ has been demonstrated to be involved in bacterial virulence. As typical bacterial phytochromes, $X c c B p h P$ covalently binds biliverdin IX $\alpha(\mathrm{BV})$ as its chromophore through the auto-catalytic bilin-lyase activity of the GAF domain, giving rise to the photosensory properties by BV-photoisomerization (20-24). When $X c c$ BphP is irradiated with far-red light, it photoconverts to the Pr form, while red light irradiation gives rise to a mixture of $\operatorname{Pr}$ and $\operatorname{Pfr}(\sim 2: 1$ with a $630-\mathrm{nm}$ light source). In XccBphP, dark conversion leads to a further enrichment of Pfr, reaching a Pfr:Pr proportion of $\sim 6: 1$ at equilibrium; we therefore have classified $X c c \mathrm{BphP}$ as a bathy-like phytochrome (17). We have previously solved the crystal structure of the full-length $X c c B p h P$ in the Pr state (PDB code: 5AKP) (17), which harbours the PAS2-GAF-PHY domain triad as its photosensory module and a PAS9 output domain (Figure 1). However, the Pfr structure remains elusive. In order to generate a more complete picture of the structural changes that occur during the Pr-Pfr transition, the structure of its Pfr is needed.

\section{$<$ Figure 1 $>$}

The aims of this work are the development of an efficient screening methodology for the rapid characterization of phytochrome variants and the identification of variants in $X c c B p h P$ that favour the Pfr form. These variants could become valuable tools in our attempt to obtain crystal structures of $X c c \mathrm{BphP}$ in the Pfr conformation. Here, we present a methodology for a rapid spectroscopic characterization of phytochrome variants. The screening approach consists of analyzing the UV-Vis absorption behaviour of clarified crude extracts from Escherichia coli overexpressing recombinant proteins in microplates. This strategy has allowed us to (i) quickly explore over a hundred of $X c c \mathrm{BphP}$ variants, (ii) characterize their photochemistry and (iii) identify Pfr-favoured candidates for future studies. This method could in principle 
be easily adapted and applied to other phytochromes or other photoreceptor families to find variants with altered photochemical behaviour.

\section{MATERIALS AND METHODS}

\section{Amino acid frequency analysis}

Sequences from the UniProtKB database were searched against the PHY domain HMM profile retrieved from Pfam database (https://pfam.xfam.org/, family PF00360) using the hmmsearch program in the EBI server (https://www.ebi.ac.uk/Tools/hmmer/) and default parameters (Input file: PHY.hmm; Output file: UniProtKB_IDs.txt). The 15,1931 positive UniProtKB AC/ID entries were mapped to the UniRef50 database (clustering homologs to reduce redundancy) using the online mapping tool provided online by the EBI server (https://www.uniprot.org/uploadlists/) to reduce the number of UniProtKB redundant and highly similar sequences. The 1,190 resulting mapped UniRef50 entries were downloaded in a FASTA format file (Input file: UniProtKB_IDs.txt; Output file: UniRef50.fasta). The UniRef50 FASTA sequences were scanned locally against PAS2, GAF and PHY domain HMM profiles retrieved from Pfam database (families PF08446, PF01590 and PF00360, respectively) using HMMER 3.2 (http://hmmer.org/download.html) (Input files: UniRef50.fasta, PAS_2.hmm, GAF.hmm, PHY.hmm; Output file: TableDom_UniRef50.out). 750 sequences that exhibit a PAS2-GAF-PHY domain architecture were identified and their respective GAF domain sequences extracted in FASTA format (Input files: TableDom_UniRef50.out, UniRef50.fasta; Output file: BphP_UniRef50_GAF.fasta). A multiple sequence alignment (MSA) was performed using Clustal-O program in the EBI server (https://www.ebi.ac.uk/Tools/msa/clustalo/) and default parameters of $751 \mathrm{GAF}$ domains retrieved from UniRef50 and XC_4241 (XccBphP) sequences (Input file: BphP_UniRef50_GAF.fasta; Output file: clustalo-GAF_UniRef50.clustal_num). The XccBphP L193 homolog residue at the MSA was analyzed for amino acid frequency.

Protein sequences were analyzed using custom MATLAB (version 2015a) scripts. The results are shown in Table S1 and all files mentioned in the above paragraph are provided as Supporting Information.

\section{Site directed mutagenesis and library construction}

First, using the pET24a-6xHis- $X c c B p h P$ full-length vector (25) as template, the PG-WT short version was generated amplifying the sequence corresponding to the region spanning from residues 1 to 313 of $X c c B p h P$ (using primers T7 + XccBphP_313_R). Then, the pET24a backbone was amplified in two separated amplicons (using primers pET24a_Gibson_NdeI_F + pET24a_Gibson_KmR_F and pET24a_Gibson_HindIII_R + pET24a_Gibson_KmR_R). Finally, the three PCR fragments were assembled together using the Gibson assembly protocol (26) into a new vector named pET24a-6xHis-PG-

This article is protected by copyright. All rights reserved 
WT. All point mutant constructs were generated using pET24a-6xHis- $X c c B p h P$ as template, through a Gibson assembly approach. Briefly, two independent amplicons, with 3' and 5' complementary regions, were generated from the template using specific primers (Table S2, one set of primers bearing the specific mutation, while the other corresponds to the kanamycin cassette on the vector backbone). Then, both PCR fragments were assembled following Gibson protocol, where only whole reconstituted vectors were able to transform E. coli DH1OB. The resulting constructs were verified by DNA sequencing. Similarly, the G454X library was generated using degenerate primers corresponding to the residue position 454 for the first amplicon generation, then the procedure followed as described above. Sanger's DNA sequencing chromatograms corroborated the expected variation at this position (Figure S1).

\section{Protein overexpression and purification}

Protein-enriched cell lysates used in the screening were obtained as follows. E. coli BL21(DE3)pLysS cells transformed with pET-24a-XccBphP constructs were grown overnight in $50 \mathrm{ml}$ of LB medium with $25 \mu \mathrm{g}$ $\mathrm{ml}^{-1}$ kanamycin and $17 \mu \mathrm{g} \mathrm{ml}^{-1}$ chloramphenicol at $37{ }^{\circ} \mathrm{C}$ in a $250 \mathrm{ml}$ Erlenmeyer flask with agitation (220 r.p.m.). Cultures were then diluted 100-fold, grown to an optical density at $600 \mathrm{~nm}$ of 0.6 and induced by the addition of $1 \mathrm{mM}$ isopropyl $\beta$-D-1-thiogalactopyranoside (IPTG) final concentration. Cultures were further incubated for $16 \mathrm{~h}$ at $20^{\circ} \mathrm{C}$ with agitation (220 r.p.m.) and then centrifuged at 9,800 x $g$ for 15 min at $4{ }^{\circ} \mathrm{C}$. Bacterial pellets were resuspended in one tenth of their original culture volume and sonicated in 50 $\mathrm{mM}$ Tris- $\mathrm{HCl} \mathrm{pH} 7.5,0.25 \mathrm{M} \mathrm{NaCl}$. Lysates were then clarified by two rounds of centrifugation at $21,000 \mathrm{x}$ $g$ for $30 \mathrm{~min}$ at $4{ }^{\circ} \mathrm{C}$ and the supernatant fractions obtained were used as apoprotein sources. Coomassiestained sodium dodecyl sulfate-polyacrylamide gel electrophoresis (SDS-PAGE) was used to assay protein levels (Figure S2).

The purification of wild-type (WT) XccBphP holoprotein was performed as previously described (17, 25). Holoproteins were produced by incubation of purified apoproteins for $1 \mathrm{~h}$ in absence of light at room temperature. Further purification steps were performed under dim green-filtered light (band-pass filter maximum at $525 \mathrm{~nm}$ ), minimizing $X c c B$ phP excitation.

\section{UV-Vis spectra measurements}

The screening measurements were performed in a Thermo Scientific Multiskan GO Microplate Spectrophotometer using Thermo Scientific Multiskan GO Microplate Spectrophotometer 96-multiwell plates (Thermo Fisher Scientific). This equipment allows the simultaneous measurement of UV-Vis spectra in 96-well plates in thermostatic and dark conditions and with programmed sampling. The volume of cell lysate used for each well was $200 \mu \mathrm{l}$. After the addition of BV (Sigma-Aldrich, product number 30891), the supernatant fractions were incubated at $25{ }^{\circ} \mathrm{C}$ in darkness for $20 \mathrm{~min}$. As a reference, after a 5-min incubation with $\mathrm{BV}$, the holo- $X c c \mathrm{BphP}$ is completely assembled (17). We chose the time of $20 \mathrm{~min}$ as a 
trade-off between maximizing the BV assembly and minimizing the total time that the clarified crude extracts are at non-refrigerated temperatures to avoid denaturation/precipitation/proteolysis phenomena. For the $2 \mathrm{~h}$ experiments (Figures 3-4 and Table 2 and Table S3), the samples were then irradiated with red (625 $\mathrm{nm}$ ) light at $0.2 \mu \mathrm{mol} \mathrm{m} \mathrm{m}^{-2} \mathrm{~s}^{-1}$ fluence for $20 \mathrm{~min}$ and their UV-Vis spectra acquired at 0,1 and $2 \mathrm{~h}$ postirradiation between 240 and $850 \mathrm{~nm}$ in the dark. After the $2 \mathrm{~h}$ incubation in the dark, the samples were irradiated with far-red light $(733 \mathrm{~nm})$ at a fluence $0.6 \mu \mathrm{mol} \mathrm{m}^{-2} \mathrm{~s}^{-1}$ for $7 \mathrm{~min}$ and final UV-Vis spectra were acquired. For the $\sim 16 \mathrm{~h}$ experiment (Figures 5-7 and Table 3), the samples from the G454X library were irradiated with far-red $(733 \mathrm{~nm})$ light at $0.6 \mu \mathrm{mol} \mathrm{m} \mathrm{m}^{-2} \mathrm{~s}^{-1}$ fluence for $7 \mathrm{~min}$ and their UV-Vis spectra acquired at 0 to 970 min post-irradiation between 240 and $850 \mathrm{~nm}$ in the dark. The sampling times were the following: $0,5,10,15,20,25,30,35,40,45,50,55,60,80,100,130,190,250,310,370,490,610,730$, 850 and $970 \mathrm{~min}$. The sequence of temporal events corresponding to light treatments and measurements of both the 2-h and the 16-h experiments are shown in panel A of Figures 3-5. For purified WT, UV-Vis absorption spectra were collected for $17 \mathrm{~h}$ at $20.0 \pm 0.2^{\circ} \mathrm{C}$ on a Cary60 UV-Vis spectrometer (Agilent) with an installed temperature control Peltier module. The sample was illuminated with far-red light at a fluence of $0.6 \mu \mathrm{mol} \mathrm{m} \mathrm{m}^{-2} \mathrm{~s}^{-1}$ for $7 \mathrm{~min}$ prior to the measurements in the dark. The protein concentration was $14 \mu \mathrm{M}$ in $50 \mathrm{mM}$ Tris $\mathrm{pH} 7.5,0.25 \mathrm{M} \mathrm{NaCl}$.

\section{UV-Vis spectra analysis}

All the steps of the analysis were performed using custom made pipelines developed in MATLAB software (version 2015b). For each G454X variant, the UV-Vis datasets consisted of 25 spectra acquired from 0 to 970 min. For visualization in Figure 2 for each variant, the minimum values were subtracted and then each spectrum was normalized to the maximum value in the dataset in the 540-850-nm range. As an observation, it can be seen in Figure 2 that three variants (G454R, G454H and G454D) seem to present an incomplete BV assembly by the time the dark conversion begins. Due to the high background in the near UV and blue regions, the datasets were processed and trimmed to the 540-850-nm range). For each spectrum, a linear baseline fit (non-zero slope) was calculated and subtracted, then normalized to the highest value in the range (Figure 3). In each processed dataset, the spectrum that exhibited the highest Abs688nm / Abs752nm relation (corresponding to spectra close to time $0 \mathrm{~min}$, immediately after far-red irradiation) was smoothed and selected to act as the corresponding pure Pr spectral component (pure-Pr). The pure Pfr spectral component (pure-Pfr) estimation was obtained by performing the subtraction of a multiple of pure-Pr from the last spectrum acquired in the dataset, that best approximates to a straight line in a selected range (a 100 $\mathrm{nm}$ variable window situated $20 \mathrm{~nm}$ towards lower wavelengths from the detected Pr $\lambda$ max peak, situated between 562 and $670 \mathrm{~nm}$ ). Although this approximation does not take into account the vibronic signatures of Pr and Pfr and therefore may not be fully exact, the fact that it was applied to all datasets in the same fashion, make the kinetics results from the $X c c \mathrm{BphP}$ variants comparable. 
$<$ Figure 2>

$<$ Figure $3>$

Then, using the pure-Pr and pure-Pfr spectra for each variant, a double Gaussian fit was performed for the peaks at around 682 and $752 \mathrm{~nm}$ and the wavelengths at which their corresponding maxima were determined (Pr- and Pfr- $\lambda$ max, Table 3 ). We then calculated the linear combinations of pure-Pr and purePfr to fit the datasets. This allowed us to obtain the individual Pr and Pfr spectra that added together, reconstitute the dataset. In Figure S5 we show the last spectra recorded at minute 970 and their respective individual Pr and Pfr estimated spectral components. The Pr and Pfr abundance (\%) at each time was estimated as the relative spectral area corresponding to pure-Pr and pure-Pfr with respect to the total spectral area (Figure 4).

$<$ Figure 4>

The kinetic analysis was carried out by obtaining the optimal coefficients A to E of equation 1 (Eq. 1) to fit the Pr or Pfr abundance data as a function of time. Using the fitted coefficients, the Pr and Pfr abundance at equilibrium (coefficient A) and a Pr/Pfr conversion rate, which we named half-life' (the time at which the absolute difference between the equilibrium and initial abundances has reached half its value, similar to the half-life parameter derived from a monoexponential function) were estimated (Table 3).

\section{$f(t)=A+B \cdot e^{\frac{-t}{C}}+D \cdot e^{\frac{-t}{E}} \quad$ (Eq. 1$)$}

A similar pipeline was applied to the purified wild-type presented in Figure 5 where all of the steps mentioned above were applied with the exception of the UV-Vis spectrum trimming and linear baseline subtraction.

$<$ Figure 5 $>$

Each processed dataset is graphically shown in individual GIF files available in Supporting Information, overlaying individual pure Pr and Pfr spectral components, and their relative abundances at each time point.

\section{RESULTS}

\section{Design of $X c c B p h P$ variants}

We designed a series of variants in $X c c B p h P$ based on (i) the spectroscopic behaviour described in other phytochromes, (ii) the analysis of the structure of $X c c \mathrm{BphP}$ in the Pr state and (iii) the analysis of amino acid conservation and frequency within the GAF domain of the phytochrome family (Table 1 and Figure 1). 
The chosen homologous mutations reported to affect the photochemistry of other phytochromes comprise the subtitutions D199A, V266S and L193 (various substitutions, detailed below) in the GAF domain (2729), and S474E and G454E in the tongue region of the PHY domain (29). The double subtitution L193CF475C was designed to favour the Pfr conformation by crosslinking through a covalent disulfide bridge between the positions 193 and 475, which are distant in space in the Pr form and should, in principle, be facing each other in the Pfr state, as judged by the conformation of the homologous positions in the crystal structure of Pseudomonas aeruginosa BphP (27, 28). Finally, a series of variants at the position 193 of $X c c B p h P$ were chosen, according to their natural frequency in the homologous position within the GAF domain from the phytochrome family that contains the PAS2-GAF-PHY domain triad (Table S1). The photochemistry of all these variants mentioned above was characterized by a rapid screening method, which is the central theme of this article.

\section{$<$ Table 1>}

Additionally, based on previous works which have generated high-resolution crystal structures of other phytochromes (30-36), and reports that propose that the PHY domain plays a key role in modulating the Pr-Pfr photochemistry $(6,37,38)$, two short constructs were designed composed of only the PAS2 and GAF domains (containing residues 1 to 313), namely PG-WT and PG-D199A. With the aim to use these constructs to obtain high-resolution structures, we decided to evaluate their photochemical behaviour previous to crystallogenesis and included them in the screening described below.

\section{Screening settings}

The method takes advantage of the fact that the E. coli clarified extracts do not considerably absorb light in the visible range. Briefly, it consists of overexpressing the XccBphP variants in E. coli BL21(DE3)pLysS, lysing the cell cultures, incubating in the dark with an appropriate amount of BV for holoprotein assembly and measuring the UV-Vis spectra (Figure 6). The UV-Vis spectra of all the $X c c B p h P$ variants cell lysates were acquired simultaneously in a microplate spectrophotometer.

$<$ Figure 6>

The volume of cell lysate needed for the experiment is relatively small $(200 \mu \mathrm{l})$, and for this reason we explored scaling down the volume of the $X c c \mathrm{BphP}$ overexpressing cultures relative to our standard affinity tagging purification protocol. The initial idea was to perform this step in a culture type 96 -well plates ( $2 \mathrm{ml}$ per well). However, protein expression levels were below the sensitivity threshold for the screening. Then, we assayed gradually incrementing cultures volumes from 5 to $50 \mathrm{ml}$, using $50-\mathrm{ml}$ falcon-type plastic tubes (5-10 $\mathrm{ml}$ cultures) and Erlenmeyer flasks (10-50 ml cultures) (Figure S3, left panel). The minimal culture volume with appropriate protein expression levels selected was $25 \mathrm{ml}$ in a $250-\mathrm{ml}$ Erlenmeyer flask for 
WT. Regarding reproducibility, culture volumes of $50 \mathrm{ml}$ in $250-\mathrm{ml}$ Erlenmeyers proved to be the more effective ones for various variants tested, therefore, this condition was chosen for the screening.

Free BV presents a broad and low intensity absorption peak around $660 \mathrm{~nm}$ and upon holoprotein assembly it gives rise to narrower and more intense peaks, namely the $\mathrm{Q}$ bands $(25,39)$. If free BV concentration is relatively high, this broad peak can mask the holo-XccBphP signal due to spectra overlap. In order to optimize the holoprotein signal, we lowered the amount of BV by minimizing free-BV signal while maintaining holoprotein signal as high and clean as possible (Figure S3, right panel). The BV final concentration selected for the screening was $21.9 \mu \mathrm{M}$.

\section{Screening results}

After optimizing the screening conditions, the selected $X c c B p h P$ variants mentioned above were assayed. The samples were incubated in the dark for at 0,1 and $2 \mathrm{~h}$ after irradiation with red light from a LED source $(625 \mathrm{~nm})$. The red light, which generates a Pfr:Pr mixture (ratio $\sim 2: 1$ in WT) allows for the identification of either Pr or Pfr enrichment (17). Then, at the end of the incubation, the samples were irradiated with far-red light to photoconvert them to the Pr state (Figures 3-4, panel A). All of them exhibited dark assembly between apoproteins and BV into holoproteins in the Pr state (Figure 7B and Table 2).

\section{$<$ Figure 7 $>$ \\ $<$ Table 2>}

From the comparison of the spectra after $1 \mathrm{~min}$ and 148 min post-incubation, it can be observed that in most variants the amount of the $\mathrm{Pr}$ is increased, indicating that the BV assembly is partial after 1 min. Apparently, the rate of BV assembly varies dramatically among the variants (Figure 7B). Additionally, all the variants present an altered photocycle compared to the wild-type (Figure 8B and Table 2). Several mutations resulted in Pr-locked versions of $X c c B p h P$, including the truncated versions PG-WT and PGD199A, comprising only PAS2 and GAF domains (Table 2). D199A and V266S substitutions also generated Pr-locked species (Table 2). This was not unexpected for D199A as the homologous mutation performed in $P a B p h P$ resulted in a Pr-locked state (27). This Pr-locking behaviour can be explained by the elimination of the stabilizing contact between D199 and BV ring-D in the Pfr form (PDB entry 3C2W) (27). Interestingly, the substitution in the $X c c \mathrm{BphP}-\mathrm{V} 266 \mathrm{~S}$ homologous position for $P a \mathrm{BphP}$ (S261A) results in a strong stabilization of its Pfr state due to the elimination of the interaction between the Ser residue and the propionate group from BV ring-C established in the Pr conformation (PDB entry 3G6O) (28). Following the same rationale, the addition of a Ser residue in XccBphP-V266S might be providing stronger contacts with the ring-C propionate and a stronger stabilization of its Pr state, preventing the Pr-Pfr photoconversion.

$<$ Figure 8> 
Strikingly, the L193C-F475C variant not only failed in locking XccBphP in Pfr as intended, but resulted in the exact opposite behaviour, locking it in the Pr state (Figure 8B). To check whether this outcome was due to formation of disulfide bridges, we incubated the XccBphP-L193C-F475C protein extracts in reducing conditions, using dithiothreitol (DTT), obtaining the same Pr-locked species (Figure S4). We then sought to find a point substitution in the L193 position as this presents a natural non-random variability among distant orthologs (Table S1). It is then possible that different side chains in homologous positions of L193 are fine-tuning the photochemistry in other phytochromes, as evidenced in other reports (27), and that their conservation might be a proxy for relevance. Based on these assumptions, we selected the residues to replace L193 taking into account their frequency and side-chain group type (Table S1). Analyzing the mutational results corresponding to the L193 position, all six selected substitutions presented a $\operatorname{Pr}$ enrichment (Table 2), indicating that this Leu residue is likely stabilizing the $\mathrm{Pfr}$ in $X c c \mathrm{BphP}$ and that it cannot be easily replaced by any other residues and still retain the Pfr-stabilizing effect. Thus, it is possible that the L193C change in XccBphP-L193C-F475C is sufficient for locking it in Pr, however, this was not further investigated in this work and remains to be confirmed.

A 2-h dark conversion experiment was enough to observe the stabilization behaviour towards a photostate, where most variants did not appear to have reached equilibrium (Figure 8B). A particular case was $X c c B p h P-G 454 E$, which seems to have almost reached equilibrium by the end of the experiment, highly enriched in Pfr. All Pfr-stabilizing variants found, including XccBphP-G454E, are not locked, as the far-red irradiation generates the photoconversion back to the Pr state (Figure 7B). We then sought to evaluate whether other substitutions in the position 454 would achieve greater Pfr stabilizations or even Pfr-locking, by constructing a random mutagenesis library (G454X).

\section{Library G454X}

The library consists of a mixture of pET24a-XccBphP recombinant plasmids degenerated at the codon corresponding to the 454 position. To generate protein producing clones, E. coli BL21(DE3)pLysS were transformed with 100, 10 and 1, ng of DNA, yielding numerous colonies in three cases. To maximize the chance of transforming cells with a single recombinant plasmid, plates derived from the lowest amount of DNA (1 ng) were chosen to continue the screening. 192 colonies were transferred to two 96-well microplates, grown in LB-10\% glycerol liquid media at $37{ }^{\circ} \mathrm{C}$ with agitation $(220$ r.p.m.) for $16 \mathrm{~h}$ and stored at $-80{ }^{\circ} \mathrm{C} .96$ colonies ( 48 from each plate) were selected for the further characterization. The culture growth, the recombinant $X c c B p h P-G 454 X$ induction, the clarified crude extract preparation, the BV addition and the $2 \mathrm{~h} \mathrm{UV-Vis} \mathrm{measurements} \mathrm{were} \mathrm{performed} \mathrm{as} \mathrm{detailed} \mathrm{for} \mathrm{the} \mathrm{previous} \mathrm{experiment} \mathrm{in}$ Figures 2 and panel A from Figures 3-4. Then, 48 of these clones, which showed good spectral quality, were sequenced. 18 substitutions, including the wild-type version, out of the 20 expected substitutions, 
were represented in the sequenced clones; only G454M and G454K were missing. All of the variants exhibited similar bathy-like photochemical behaviour. They were all unlocked, photo-converting upon, both, red and far-red light (Table S3).

In order to deepen the photochemical characterization of these variants, we performed an experiment in which their corresponding clarified crude extract samples were supplemented with BV and illuminated with far-red light $(733 \mathrm{~nm}$ ) during $7 \mathrm{~min}$ (taking into account they all present bathy-like behaviour). Then, the dark conversion was monitored by acquiring the UV-Vis spectra for $\sim 16 \mathrm{~h}$ (Figure 2). The UV-Vis datasets were then further processed (Figure 3) and the temporal evolution of Pr and Pfr was fitted to a biexponential function (Figure 4). Three of the variants (G454R, G454H and G454D) seem to present an incomplete BV assembly by the time the dark conversion begins, as evidenced by an increase in the Pr form after the initial time (Figure 2). For the variants presenting complete BV assemblies, kinetic rates and equilibrium abundances were calculated (Table 3). The kinetic rate was estimated as the time at which the absolute difference between the equilibrium and initial abundances reached half its value, similar to the half-life parameter derived from a monoexponential function and therefore named half-life'.

$<$ Table 3>

In these clarified crude extract experiments, the wild-type version presented a similar behaviour to the one already reported (17), with a Pfr proportion at the equilibrium value of $\sim 78 \%$ and a half-life' of $\sim 309$ min (Table 3). Interestingly, only the G545I variant presented a similar behaviour to the wild-type. The rest of the characterized substitutions presented a Pfr abundance value at the equilibrium greater than $90 \%$ with half-lives' ranging from 24 to $300 \mathrm{~min}$. There were no substantial differences between the wild-type and the mutants in the wavelengths corresponding to the Pr or Pfr maxima ( $\lambda$ max, Table 3 ). In order to rank the mutant versions according to their propensity towards the Pfr state, a score was calculated dividing the Pfr abundance at equilibrium by the half-life' (Table 3), where G454E presents the highest score, with a Pfr abundance value at equilibrium of $\sim 99 \%$ and a half-life' value of $\sim 24 \mathrm{~min}$.

\section{Validation of the screening approach using the wild-type $X c c B p h P$ purified protein}

As already mentioned, the results of $X c c$ BphP Pfr proportion at the equilibrium $(\sim 78 \%)$ and half-life' value ( $\sim 308 \mathrm{~min}$ ) obtained in clarified crude extract experiments were very similar to those already reported for the purified holoprotein ( $\sim 85 \%$ and $\sim 447 \mathrm{~min}$, respectively). The small difference observed may reside, at least in part, in the data analysis and the experimental conditions such as temperature and the buffer composition. In order to make a better comparison and validate the screening method, we purified wildtype holo $X c c \mathrm{BphP}$, performed the $\mathrm{UV}-\mathrm{V}$ is measurements in the same experimental conditions as in clarified crude extracts experiments and subjected the data to the same data analysis treatments (Figure 5 and Table 3). The values obtained for pure protein were $\sim 72 \%$ and $\sim 315 \mathrm{~min}$, for the Pfr proportion at 
equilibrium and the half-life', respectively. These results are even in closer agreement to the ones from the screening method using $X c c B p h P$ clarified crude extract, demonstrating that this strategy is very suitable not only for qualitative, but also for quantitative characterization of phytochrome variants.

\section{DISCUSSION}

In this article we present a simple and economical strategy for the simultaneous and rapid characterization of a large number of bacteriophytochrome variants from overexpressing E.coli extracts. This method provided high-quality absorption spectra suitable not only for qualitative, but also for quantitative studies. The strategy is based on the fact that rich information of the photochemical behaviour of the photoactive proteins resides in the visible range, where bacterial extracts do not significantly absorb. This approach may be used in other systems as well, such as other phytochrome or other photoreceptor families. Furthermore, this approach was validated with purified protein obtaining very similar results, and may be easily adapted to be used in other systems.

Performing a rapid 2-h experiment, the methodology provided qualitative information about three important aspects of the photochemical behaviour from different $X c c \mathrm{BphP}$ variants. First, it allowed us to study the holoprotein assembly, determining that all the variants studied assemble, like the wild-type version, into the Pr form. Second, we could gather information about the direction of the dark conversion, allowing the classification of variants into the Pr- or Pfr-favoured categories, which were both represented among the generated variants. Finally, we were able to cluster them into slow or fast categories, according to their dark conversion rates. This characterization allowed us to identify the key position 454 located in the tongue of the PHY domain, which dramatically stabilizes the Pfr form.

Subsequently, in a 16-h experiment, quantitative information about the dark conversion process could be gathered on multiple variants simultaneously. This Pr/Pfr abundance plot along time could be modeled with an exponential equation, where the curve's plateau represents the composition at the equilibrium and the curvature or half-life describes the dark conversion rate. This approach was applied to the mutant random library G454X, obtaining 18 out of 20 possible substitutions and where all of them produced bathy-like phytochromes. Interestingly, all G454 variants present a higher Pfr proportion at equilibrium or a higher dark conversion rate than the wild-type. The fact that a wide range of residues of varied chemical nature in G454 substitutions favours the Pfr form could be indicative of a destabilization of the Pr form rather than a stabilization of the Pfr form. There is probably a tight constraint for flexibility in the Pr state at the G454 position, which is located in a loop belonging to the tongue region. However, to gain insight into the role of the G454 position, further biophysical studies need to be performed, which are beyond the scope of this work. To finish, the method presented here allowed us to identify several $X c c B p h P$ variants with extremely favoured Pfr and, therefore, good candidates for future structural studies.

This article is protected by copyright. All rights reserved 


\section{ACKNOWLEDGEMENTS}

We acknowledge the kind help and technical advice of Dr. Vanina Alzogaray on the manipulation of the G454X library. This work was supported by the Argentinian Ministry of Science (MINCyT), the Argentinian Research Council (CONICET), and the National Agency for the Promotion of Science and Technology of Argentina (ANPCyT), under the grant PICT 2016-1425.

\section{SUPPORTING INFORMATION}

Additional supporting information may be found online in the Supporting Information section at the end of the article:

Table S1. Frequency analysis of amino acid conservation from a diverse set of 748 orthologs at the corresponding homologous positions to Xcc BphP-L193.

Table S2. Oligonucleotides used in this study.

Table S3. G454X library screening. 48 of the 96 screened clones were selected for sequence determination. Not determined data (i.e. sequence or UV-Vis spectra) are annotated as "ND". The dark conversion rate classification (fast or slow) follows the same criteria as in Table 2.

Figure S1. Degenerate codon number 454 from the Xcc BphP-G454X library. The chromatogram derived from Sanger's DNA sequencing of the Xcc BphP-G454X library constructed on the pET24a-6xHis- Xcc BphP shows the codon variability at position 454 (G454). Both the DNA and the protein sequences are indicated.

Figure S2. Xcc BphP variants overexpression. $12 \%$ SDS-PAGE of clarified bacterial supernatants samples containing several over-expressed Xcc BphP variants.

Figure S3. Screening conditions optimization. Left panel: Culture volume and container determination. Different containers and culture volumes were assessed in order to determine the smallest bacterial culture in which the BphP Q bands could be discerned. 50-mL Falcon tubes (F) or 50-ml and 250-mL Erlenmeyer flasks (E50 and E250, respectively) were used to incubate 10, 25 or $50 \mathrm{~mL}$ of bacterial cultures. Excess of BV (continuous traces) was added to the clarified crude extracts obtained from each culture to induce the formation of holo- Xcc BphP. The dashed traces correspond to cell lysates with no BV added. Right panel: BV titration. An apo- Xcc BphP extract obtained in the culture condition E250-50 mL was titrated with increasing BV concentrations. 
Figure S4. Effect of reducing agent on the Xcc BphP cysteine variant L193C-F475C. Clarified Xcc BphP L193C-F475C extracts were incubated with BV and different concentrations of DTT for $20 \mathrm{~min}$. UV-Vis spectra were captured immediately after red light irradiation (0 min, dashed trace) or following a 120 min dark incubation after irradiation (continuous traces).

Figure S5. Spectral analysis from G454X variants multiwell samples. Data derives from Figure 3, example at minute 970 from UV-Vis spectra dataset processed in the 540-840-nm range (black trace) with their respective individual Pr and Pfr pure spectra estimates (fit) and their sum (dashed trace). Abundances (\%) are indicated in the references.

\section{REFERENCES}

1. Rockwell, N. C. and Lagarias, J. C. (2006) The structure of phytochrome: a picture is worth a thousand spectra. Plant Cell 18, 4-14.

2. Rockwell, N. C., Su, Y.-S. and Lagarias, J. C. (2006) Phytochrome structure and signaling mechanisms. Annu. Rev. Plant Biol. 57, 837-858.

3. Auldridge, M. E. and Forest, K. T. (2011) Bacterial phytochromes: more than meets the light. Crit. Rev. Biochem. Mol. Biol. 46, 67-88.

4. Möglich, A. (2019) Signal transduction in photoreceptor histidine kinases. Protein Science 28, 19231946. https://doi.org/10.1002/pro.3705.

5. Rockwell, N. C. and Lagarias, J. C. (2019) Phytochrome evolution in 3D: deletion, duplication, and diversification. New Phytol. https://doi.org/10.1111/nph.16240.

6. Gourinchas, G., Etzl, S. and Winkler, A. (2019) Bacteriophytochromes - from informative model systems of phytochrome function to powerful tools in cell biology. Curr. Opin. Struct. Biol. 57, 72-83.

7. Rottwinkel, G., Oberpichler, I. and Lamparter, T. (2010) Bathy phytochromes in rhizobial soil bacteria. J. Bacteriol. 192, 5124-5133.

8. Salewski, J., Escobar, F. V., Kaminski, S., von Stetten, D., Keidel, A., Rippers, Y., Michael, N., Scheerer, P., Piwowarski, P., Bartl, F., Frankenberg-Dinkel, N., Ringsdorf, S., Gärtner, W., Lamparter, T., Mroginski, M. A. and Hildebrandt, P. (2013) Structure of the Biliverdin Cofactor in the Pfr State of Bathy and Prototypical Phytochromes. J. Biol. Chem. 288, 16800-16814.

9. Murgida, D. H., von Stetten, D., Hildebrandt, P., Schwinté, P., Siebert, F., Sharda, S., Gärtner, W. and 
Mroginski, M. A. (2007) The chromophore structures of the Pr States in plant and bacterial phytochromes. Biophys. J. 93, 2410-2417.

10. Siegelman, H. W. and Firer, E. M. (1964) PURIFICATION OF PHYTOCHROME FROM OAT SEEDLINGS. Biochemistry 3, 418-423.

11. Siegelman, H. W. and Hendricks, S. B. (1965) Purification and properties of phytochrome: a chromoprotein regulating plant growth. Fed. Proc. 24, 863-867.

12. Mumford, F. E. (1966) Studies on the phytochrome dark reaction in vitro. Biochemistry 5, 522-524.

13. Wahleithner, J. A., Li, L. M. and Lagarias, J. C. (1991) Expression and assembly of spectrally active recombinant holophytochrome. Proc. Natl. Acad. Sci. U. S. A. 88, 10387-10391.

14. Gärtner, W., Hill, C., Worm, K., Braslavsky, S. E. and Schaffner, K. (1996) Influence of expression system on chromophore binding and preservation of spectral properties in recombinant phytochrome A. Eur. J. Biochem. 236, 978-983.

15. Mozley, D., Remberg, A. and Gärtner, W. (1997) Large-scale generation of affinity-purified recombinant phytochrome chromopeptide. Photochem. Photobiol. 66, 710-715.

16. Hughes, J., Lamparter, T., Mittmann, F., Hartmann, E., Gärtner, W., Wilde, A. and Börner, T. (1997) A prokaryotic phytochrome. Nature 386, 663.

17. Otero, L. H., Klinke, S., Rinaldi, J., Velázquez-Escobar, F., Mroginski, M. A., Fernández López, M., Malamud, F., Vojnov, A. A., Hildebrandt, P., Goldbaum, F. A. and Bonomi, H. R. (2016) Structure of the Full-Length Bacteriophytochrome from the Plant Pathogen Xanthomonas campestris Provides Clues to its Long-Range Signaling Mechanism. J. Mol. Biol. 428, 3702-3720.

18. Bonomi, H. R., Toum, L., Sycz, G., Sieira, R., Toscani, A. M., Gudesblat, G. E., Leskow, F. C., Goldbaum, F. A., Vojnov, A. A. and Malamud, F. (2016) Xanthomonas campestris attenuates virulence by sensing light through a bacteriophytochrome photoreceptor. EMBO reports 17, 1565-1577. https://doi.org/10.15252/embr.201541691.

19. Losi, A., Bonomi, H. R., Michael, N., Tang, K. and Zhao, K.-H. (2017) Time-Resolved Energetics of Photoprocesses in Prokaryotic Phytochrome-Related Photoreceptors. Photochem. Photobiol. 93, 733-740.

20. Lagarias, J. C., Clark Lagarias, J. and Rapoport, H. (1980) Chromopeptides from phytochrome. The structure and linkage of the PR form of the phytochrome chromophore. J. Am. Chem. Soc. 102, 4821-4828. 
https://doi.org/10.1021/ja00534a042.

21. Lamparter, T., Michael, N., Caspani, O., Miyata, T., Shirai, K. and Inomata, K. (2003) Biliverdin binds covalently to agrobacterium phytochrome Agp1 via its ring A vinyl side chain. J. Biol. Chem. 278, 3378633792 .

22. Quest, B. and Gärtner, W. (2004) Chromophore selectivity in bacterial phytochromes: dissecting the process of chromophore attachment. Eur. J. Biochem. 271, 1117-1126.

23. Tasler, R., Moises, T. and Frankenberg-Dinkel, N. (2005) Biochemical and spectroscopic characterization of the bacterial phytochrome of Pseudomonas aeruginosa. FEBS J. 272, 1927-1936.

24. Lagarias, J. C. and Lagarias, D. M. (1989) Self-assembly of synthetic phytochrome holoprotein in vitro. Proc. Natl. Acad. Sci. U. S. A. 86, 5778-5780.

25. Klinke, S., Otero, L. H., Rinaldi, J., Sosa, S., Guimarães, B. G., Shepard, W. E., Goldbaum, F. A. and Bonomi, H. R. (2014) Crystallization and preliminary X-ray characterization of the full-length bacteriophytochrome from the plant pathogen Xanthomonas campestris pv. campestris. Acta Crystallogr. Sect. F Struct. Biol. Cryst. Commun. 70, 1636-1639.

26. Gibson, D. G., Young, L., Chuang, R.-Y., Venter, J. C., Hutchison, C. A., 3rd and Smith, H. O. (2009) Enzymatic assembly of DNA molecules up to several hundred kilobases. Nat. Methods 6, 343-345.

27. Yang, X., Kuk, J. and Moffat, K. (2008) Crystal structure of Pseudomonas aeruginosa bacteriophytochrome: photoconversion and signal transduction. Proc. Natl. Acad. Sci. U. S. A. 105, 1471514720 .

28. Yang, X., Kuk, J. and Moffat, K. (2009) Conformational differences between the Pfr and Pr states in Pseudomonas aeruginosa bacteriophytochrome. Proc. Natl. Acad. Sci. U. S. A. 106, 15639-15644.

29. Burgie, E. S., Bussell, A. N., Walker, J. M., Dubiel, K. and Vierstra, R. D. (2014) Crystal structure of the photosensing module from a red/far-red light-absorbing plant phytochrome. Proc. Natl. Acad. Sci. U. S. A. 111, 10179-10184.

30. Wagner, J. R., Zhang, J., Brunzelle, J. S., Vierstra, R. D. and Forest, K. T. (2007) High resolution structure of Deinococcus bacteriophytochrome yields new insights into phytochrome architecture and evolution. J. Biol. Chem. 282, 12298-12309.

31. Burgie, E. S., Wang, T., Bussell, A. N., Walker, J. M., Li, H. and Vierstra, R. D. (2014) 
Crystallographic and electron microscopic analyses of a bacterial phytochrome reveal local and global rearrangements during photoconversion. J. Biol. Chem. 289, 24573-24587.

32. Lehtivuori, H., Bhattacharya, S., Angenent-Mari, N. M., Satyshur, K. A. and Forest, K. T. (2015) Removal of Chromophore-Proximal Polar Atoms Decreases Water Content and Increases Fluorescence in a Near Infrared Phytofluor. Front Mol Biosci 2, 65.

33. Woitowich, N. C., Halavaty, A. S., Waltz, P., Kupitz, C., Valera, J., Tracy, G., Gallagher, K. D., Claesson, E., Nakane, T., Pandey, S., Nelson, G., Tanaka, R., Nango, E., Mizohata, E., Owada, S., Tono, K., Joti, Y., Nugent, A. C., Patel, H., Mapara, A., Hopkins, J., Duong, P., Bizhga, D., Kovaleva, S. E., St Peter, R., Hernandez, C. N., Ozarowski, W. B., Roy-Chowdhuri, S., Yang, J.-H., Edlund, P., Takala, H., Ihalainen, J., Brayshaw, J., Norwood, T., Poudyal, I., Fromme, P., Spence, J. C. H., Moffat, K., Westenhoff, S., Schmidt, M. and Stojković, E. A. (2018) Structural basis for light control of cell development revealed by crystal structures of a myxobacterial phytochrome. IUCrJ 5, 619-634.

34. Takala, H., Lehtivuori, H. K., Berntsson, O., Hughes, A., Nanekar, R., Niebling, S., Panman, M., Henry, L., Menzel, A., Westenhoff, S. and Ihalainen, J. A. (2018) On the (un)coupling of the chromophore, tongue interactions, and overall conformation in a bacterial phytochrome. J. Biol. Chem. 293, 8161-8172.

35. Lenngren, N., Edlund, P., Takala, H., Stucki-Buchli, B., Rumfeldt, J., Peshev, I., Häkkänen, H., Westenhoff, S. and Ihalainen, J. A. (2018) Coordination of the biliverdin D-ring in bacteriophytochromes. Phys. Chem. Chem. Phys. 20, 18216-18225.

36. Auldridge, M. E., Satyshur, K. A., Anstrom, D. M. and Forest, K. T. (2012) Structure-guided engineering enhances a phytochrome-based infrared fluorescent protein. J. Biol. Chem. 287, 7000-7009.

37. Burgie, E. S., Zhang, J. and Vierstra, R. D. (2016) Crystal Structure of Deinococcus Phytochrome in the Photoactivated State Reveals a Cascade of Structural Rearrangements during Photoconversion. Structure 24, 448-457.

38. Velázquez Escobar, F., Buhrke, D., Michael, N., Sauthof, L., Wilkening, S., Tavraz, N. N., Salewski, J., Frankenberg-Dinkel, N., Mroginski, M. A., Scheerer, P., Friedrich, T., Siebert, F. and Hildebrandt, P. (2017) Common Structural Elements in the Chromophore Binding Pocket of the Pfr State of Bathy Phytochromes. Photochem. Photobiol. 93, 724-732.

39. Borucki, B., Otto, H., Rottwinkel, G., Hughes, J., Heyn, M. P. and Lamparter, T. (2003) Mechanism of Cph1 phytochrome assembly from stopped-flow kinetics and circular dichroism. Biochemistry 42, 1368413697.

This article is protected by copyright. All rights reserved 


\section{FIGURE CAPTIONS}

Figure 1. Selected positions for mutagenesis analysis mapped on the XccBphP Pr structure. The overall crystal structure (PDB code: 5AKP) is shown on the right. Each domain, PAS2, GAF, PHY (photosensory module) and PAS9 (output module), is shown with a different color. The tongue region in the PHY domain is indicated with a dashed box. The biliverdin molecule (BV) is shown as sticks. In the inset, key positions selected for mutagenesis are represented as sticks and atoms are colored according to their type.

\section{Figure 2. Dark conversion after far-red illumination variants derived from the G454X library} screening. (A) Scheme of the temporal sequence of light treatment and measurements. The highlighted measurements correspond to the ones used for the analysis of the dark conversion, shown in panel B. (B) $\mathrm{UV}-\mathrm{Vis}$ spectra from clarified extracts of overexpressing clones of the G454X library. The traces are shown in jet color code representing the time scale. The minimum values were subtracted and then each spectrum was normalized to the maximum value in the dataset in the 540-850-nm range (Q bands).

Figure 3. Processing of the UV-Vis spectra from the G454X variants. Absorption spectra from Fig. 2 were trimmed from $540 \mathrm{~nm}$ to $850 \mathrm{~nm}$ (range corresponding to the $\mathrm{Q}$ bands). Each individual spectrum in the dataset was subjected to linear baseline fit subtraction and normalization to the highest value in the selected range.

Figure 4. Kinetics of the Pr-to-Pfr dark conversion from G454X variants. Using the processed data from Fig. 3, Pr and Pfr individual abundance (\%) at each time was calculated for all variants, assuming that these two species are responsible for the absorbance changes during the experiment. Pr/Pfr abundance values were fitted to a double exponential function (eq. 1, black traces). Half-life values and Pr/Pfr abundances at equilibrium were estimated using the fitted coefficients and equation 1.

Figure 5. Dark conversion after far-red illumination of $X c c B p h P-W T$. A purified holoprotein protein sample (1 mg per $\mathrm{ml}$ ) was placed in a quartz cuvette, irradiated for $20 \mathrm{~min}$ with far-red light and then incubated in the dark. Left panel: UV-Vis spectra were recorded for a period of $1380 \mathrm{~min}$, normalized and represented in jet color code. Middle panel: Example at minute 1380 of $\mathrm{Pr}$ and Pfr abundance estimations. Right panel: $\mathrm{Pr} / \mathrm{Pfr}$ abundance values were fitted to a double exponential function (eq. 1, black traces). Half-life value and Pr/Pfr abundance at equilibrium were estimated using the fitted coefficients and equation 1 , and displayed in Table 3.

This article is protected by copyright. All rights reserved 
Figure 6. BphP screening methodology simplified scheme. The individual clones are cultured at $37^{\circ} \mathrm{C}$ in $50 \mathrm{~mL}$ of LB medium in a $250 \mathrm{~mL}$ Erlenmeyer flask to an optical density at $600 \mathrm{~nm}$ (OD) of 0.6 and protein expression is induced overnight at $20{ }^{\circ} \mathrm{C}$ with $1 \mathrm{mM}$ IPTG. Then, cells are harvested by centrifugation, lysated and clarified crude extracts are obtained after two rounds of centrifugation $(21,000 \mathrm{x}$ g). Holoprotein assembly is induced by the addition of BV (final concentration $21.9 \mu \mathrm{M}$ ) to $200 \mu \mathrm{L}$ of protein sample in dark conditions. The photochemistry of the clones is characterized via dark conversion measurements after red or far-red light irradiation.

Figure 7. BV assembly of $X c c B p h P$ variants. (A) Scheme of the temporal sequence of light treatment and measurements. The highlighted measurements correspond to the ones used for the qualitative analysis of BV assembly, shown in panel B. (B) UV-Vis spectra from clarified apoprotein extracts after the addition of BV in the dark. The black traces correspond to the spectra collected 1 min after the addition of BV (\#1), while the pink traces correspond to the measurements at $148 \mathrm{~min}$ (\#5), after far-red illumination.

Figure 8. Dark conversion after red light illumination of $X c c B p h P$ variants. (A) Scheme of the temporal sequence of light treatment and measurements. The highlighted measurements correspond to the ones used for the qualitative analysis of the dark conversion, shown in panel B. (B) UV-Vis spectra form clarified extracts. The blue, green and red traces correspond to the spectra collected $0(\# 2), 60(\# 3)$ and 120 min (\#4) incubation in the dark after the red light treatment, respectively. The minimum values were subtracted and then each spectrum was normalized to the maximum value in the dataset in the 540-850-nem range (Q bands). 
Table 1. $X c c \mathrm{BphP}$ variants used in this study.

\begin{tabular}{|c|c|c|c|c|}
\hline $\begin{array}{l}X c c B p h P \\
\text { variant }\end{array}$ & $\begin{array}{c}\text { Position in } X c c \text { BphP } \\
\text { structure }\end{array}$ & $\begin{array}{l}\text { Homologue } \\
\text { mutation }\end{array}$ & $\begin{array}{c}\text { Effect in } \\
\text { homologue } \\
\text { model }\end{array}$ & Reference \\
\hline D199A & GAF & $P a \mathrm{BphP}-\mathrm{D} 194 \mathrm{~A}$ & Locks $\operatorname{Pr}$ & $\begin{array}{c}\text { Yang, Kuk, and Moffat } \\
2008\end{array}$ \\
\hline V266S & $\begin{array}{c}\text { GAF (BV contact in } \\
\text { Pr) }\end{array}$ & $P a \mathrm{BphP}-\mathrm{S} 261 \mathrm{~A}$ & Locks Pfr & $\begin{array}{c}\text { Yang, Kuk, and Moffat } \\
2009\end{array}$ \\
\hline G454E & Tongue in $\mathrm{PHY}$ & AtPhyB-G564E & Favours Pfr & $\begin{array}{l}\text { Burgie, Bussell, et al. } \\
2014\end{array}$ \\
\hline S474E & Tongue in $\mathrm{PHY}$ & AtPhyB-S584E & Favours Pr & $\begin{array}{l}\text { Burgie, Bussell, et al. } \\
2014\end{array}$ \\
\hline L193H & \multirow{6}{*}{$\begin{array}{l}\text { GAF, BV contact in } \\
\text { the Pfr form }\end{array}$} & \multirow{6}{*}{$P a \mathrm{BphP}-\mathrm{Q} 188 \mathrm{~L}$} & \multirow{6}{*}{ Favours Pr } & \multirow{6}{*}{$\begin{array}{l}\text { Burgie, Wang, et al. } \\
\qquad 2014\end{array}$} \\
\hline L193T & & & & \\
\hline L193R & & & & \\
\hline L193M & & & & \\
\hline L193F & & & & \\
\hline L193E & & & & \\
\hline L193C-F475C & $\begin{array}{c}\text { GAF and tongue } \\
(\mathrm{PaBphP} \text { homologue } \\
\text { positions Q188-F460 } \\
\text { are close in } \mathrm{Pfr})\end{array}$ & - & - & $\begin{array}{c}\text { Yang, Kuk, and Moffat } \\
2008\end{array}$ \\
\hline PG-WT & \multirow{2}{*}{ PAS2-GAF } & DrBphP & $\begin{array}{c}\text { High resolution } \\
\text { structures }\end{array}$ & \multirow{2}{*}{$\begin{array}{l}\text { Wagner et al. } 2008 \\
\text { Burgie, Wang, et al. } \\
2014\end{array}$} \\
\hline PG-D199A & & $\operatorname{DrBphP}$ & $\begin{array}{l}\text { High resolution } \\
\text { structures }\end{array}$ & \\
\hline
\end{tabular}

This article is protected by copyright. All rights reserved 
Table 2. Results of the screening of $X c c \mathrm{BphP}$ variants.

\begin{tabular}{|c|c|c|c|c|}
\hline $\begin{array}{c}\text { XccBphP } \\
\text { variant }\end{array}$ & $\begin{array}{c}\text { Dark assembly } \\
\text { of BV }\end{array}$ & $\begin{array}{c}\text { Enrichment in } \\
\text { darkness }\end{array}$ & $\begin{array}{c}\text { Sensitivity to } \\
\text { light }\end{array}$ & Rate \\
\hline D199A & $\operatorname{Pr}$ & $\operatorname{Pr}$ & Locked & - \\
\hline V266S & $\operatorname{Pr}$ & $\operatorname{Pr}$ & Locked & - \\
\hline G454E & $\operatorname{Pr}$ & $\operatorname{Pfr}$ & Unlocked & Fast \\
\hline S474E & $\operatorname{Pr}$ & $\operatorname{Pr}$ & Unlocked & Fast \\
\hline L193H & $\operatorname{Pr}$ & $\operatorname{Pr}$ & Unlocked & Slow \\
\hline L193T & $\operatorname{Pr}$ & $\operatorname{Pr}$ & Unlocked & Slow \\
\hline L193R & $\operatorname{Pr}$ & $\operatorname{Pr}$ & Unlocked & Slow \\
\hline L193M & $\operatorname{Pr}$ & $\operatorname{Pfr}$ & Unlocked & Slow \\
\hline L193F & $\operatorname{Pr}$ & $\operatorname{Pfr}$ & Unlocked & Slow \\
\hline L193E & $\operatorname{Pr}$ & $\operatorname{Pr}$ & Unlocked & Fast \\
\hline L193C-F475C & $\operatorname{Pr}$ & $\operatorname{Pr}$ & Locked & - \\
\hline PG-WT & $\operatorname{Pr}$ & $\operatorname{Pr}$ & Locked & - \\
\hline PG-D199A & $\operatorname{Pr}$ & $\operatorname{Pr}$ & Locked & - \\
\hline
\end{tabular}

Variants insensitive and sensitive to light are named locked and unlocked, respectively.

Variants showing or not showing significant changes in their absorption spectra after $1 \mathrm{~h}$ are referred to as fast and slow, respectively. 
Table 3. Kinetic analysis for wild-type and G454X variants in dark conversion after far-red irradiation experiments. All entries derive from Figure 8 (clarified protein extracts) calculations except WT-purified (purified holoprotein) that derives from Figure 9 calculations.

\begin{tabular}{|c|c|c|c|c|c|c|}
\hline Variant & $\begin{array}{c}\text { Half-life' } \\
(\mathbf{m i n})\end{array}$ & $\begin{array}{c}\text { Pr eq. } \\
(\boldsymbol{\%})\end{array}$ & $\begin{array}{c}\text { Pfr eq. } \\
(\boldsymbol{\%})\end{array}$ & $\begin{array}{c}\text { Pr } \lambda \mathbf{m a x} \\
(\mathbf{n m})\end{array}$ & $\begin{array}{c}\text { Pfr } \lambda \mathbf{m a x} \\
(\mathbf{n m})\end{array}$ & $\begin{array}{c}\text { Pfr eq. (\%) / Half-life' }^{\prime} \\
(\mathbf{m i n})\end{array}$ \\
\hline WT-purified & 314.8 & 27.6 & 72.4 & 683.9 & 756.7 & 0.23 \\
\hline WT & 309.4 & 21.7 & 78.3 & 682.0 & 755.0 & 0.25 \\
\hline G454E & 24.1 & 0.8 & 99.2 & 687.4 & 749.8 & 4.12 \\
\hline G454A & 32.4 & 2 & 98 & 686.9 & 749.9 & 3.02 \\
\hline G454Q & 41.9 & 0.9 & 99.1 & 687.7 & 750.0 & 2.37 \\
\hline G454N & 43.4 & 1.4 & 98.6 & 687.0 & 749.9 & 2.27 \\
\hline G454S & 48.9 & 0 & 100 & 687.2 & 750.2 & 2.04 \\
\hline G454W & 61.6 & 3.2 & 96.8 & 686.9 & 753.1 & 1.57 \\
\hline G454C & 64.8 & 4.3 & 95.7 & 687.1 & 751.4 & 1.48 \\
\hline G454T & 70.7 & 3.1 & 96.9 & 688.0 & 749.5 & 1.37 \\
\hline G454V & 71.3 & 2 & 98 & 687.0 & 753.4 & 1.37 \\
\hline G454L & 73.8 & 0.8 & 99.2 & 689.1 & 748.9 & 1.34 \\
\hline G454F & 113.1 & 6.3 & 93.7 & 688.6 & 751.9 & 0.83 \\
\hline G454P & 151.7 & 8.3 & 91.7 & 687.4 & 751.9 & 0.60 \\
\hline G454Y & 171.8 & 8.4 & 91.6 & 687.7 & 753.4 & 0.53 \\
\hline G454I & 297.3 & 18.1 & 81.9 & 682.2 & 757.1 & 0.28 \\
\hline
\end{tabular}

This article is protected by copyright. All rights reserved 


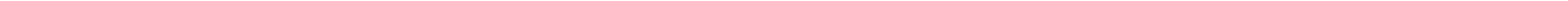


$\begin{array}{ll}100 & \text { WT }\end{array}$
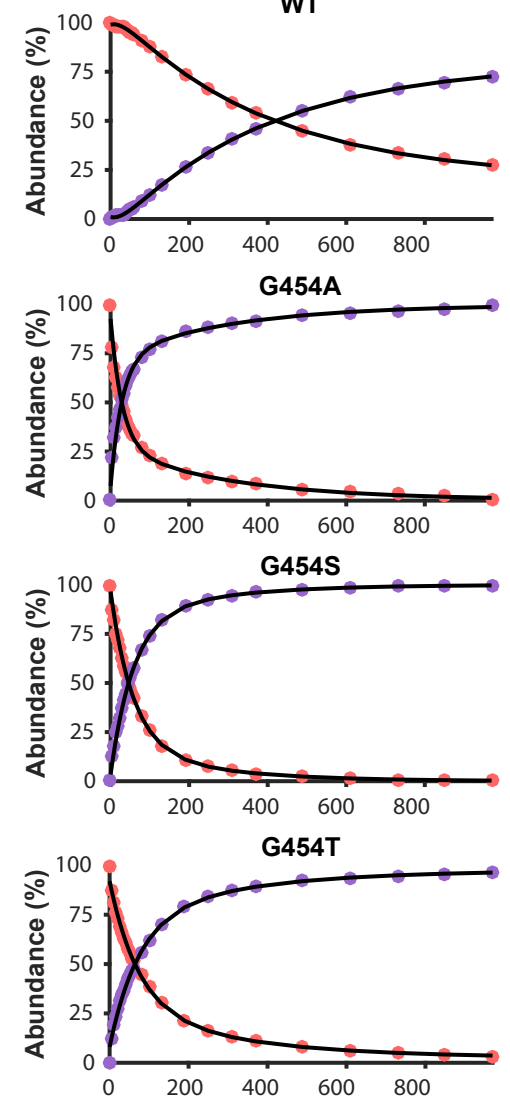

\%

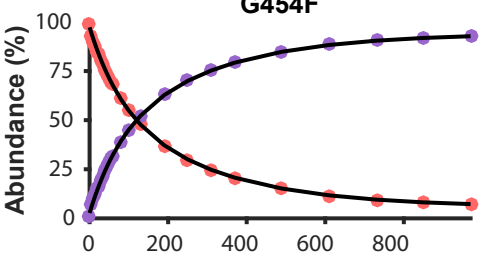

ฮे

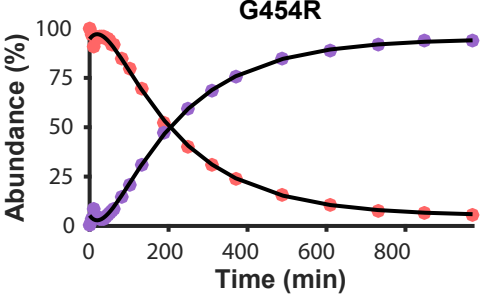

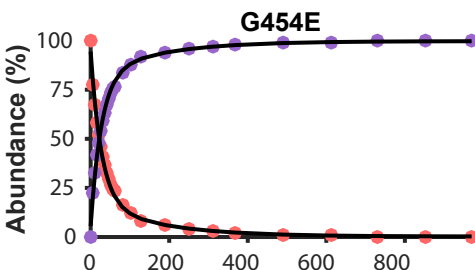
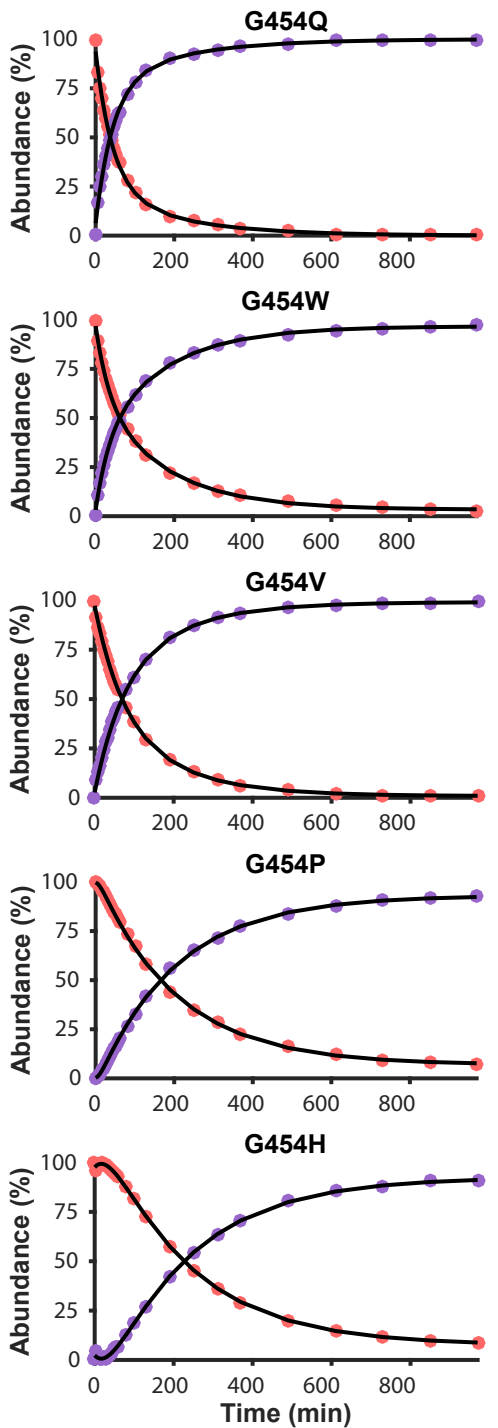
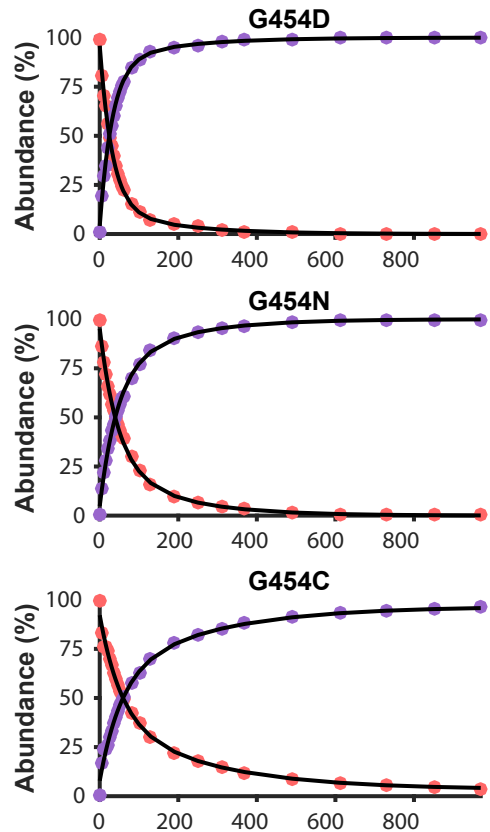

G454L

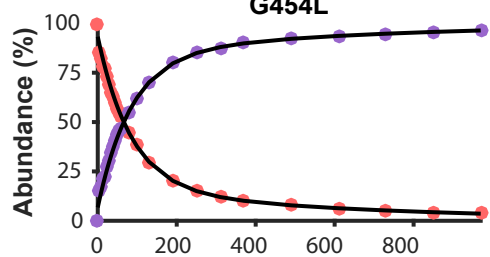

G454Y

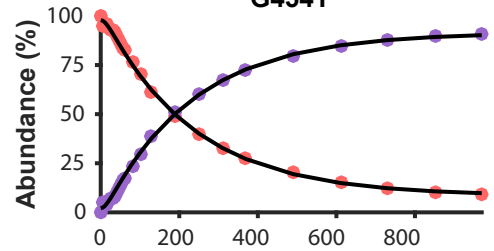

G454I

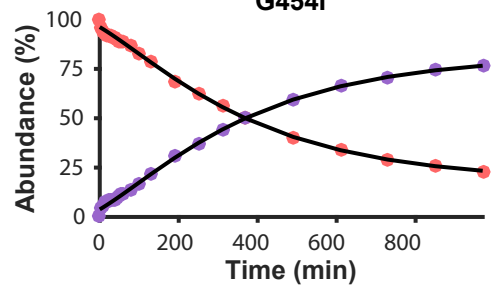

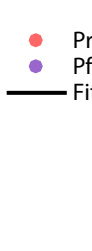
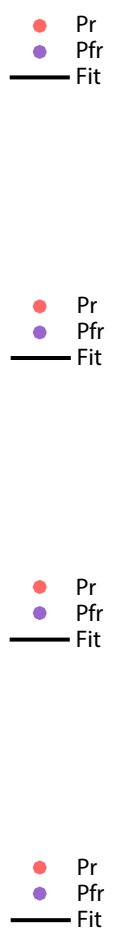

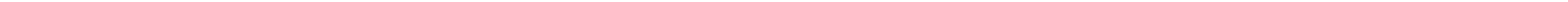


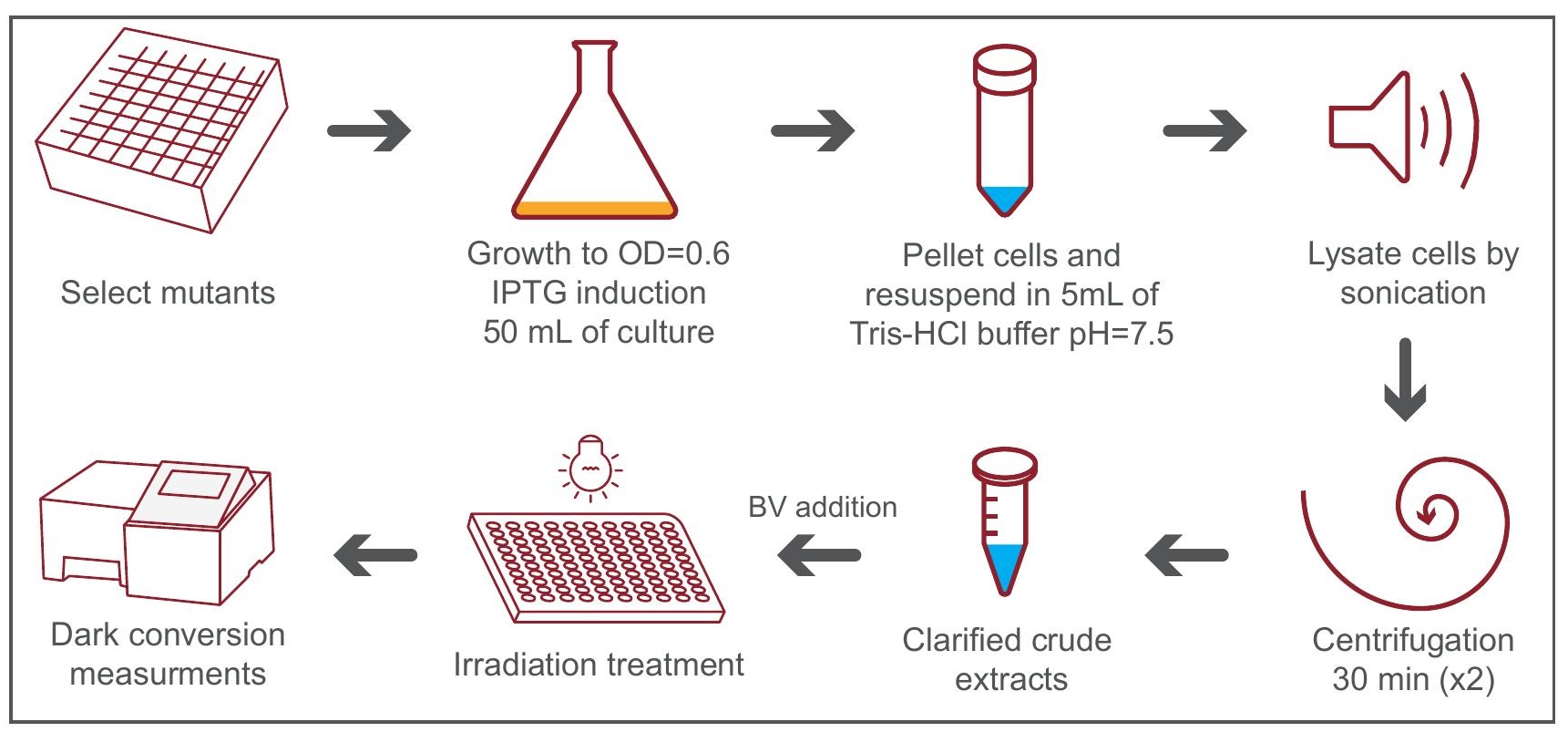


BV addition

Dark (1 min)

red light (20 min)

Dark (120 min)

far-red light (7 min)

time

measurement \#1

$\begin{array}{ccc}0 \min & 60 \min & 120 \min \\ \# 2 & \# 3 & \# 4\end{array}$

\#5
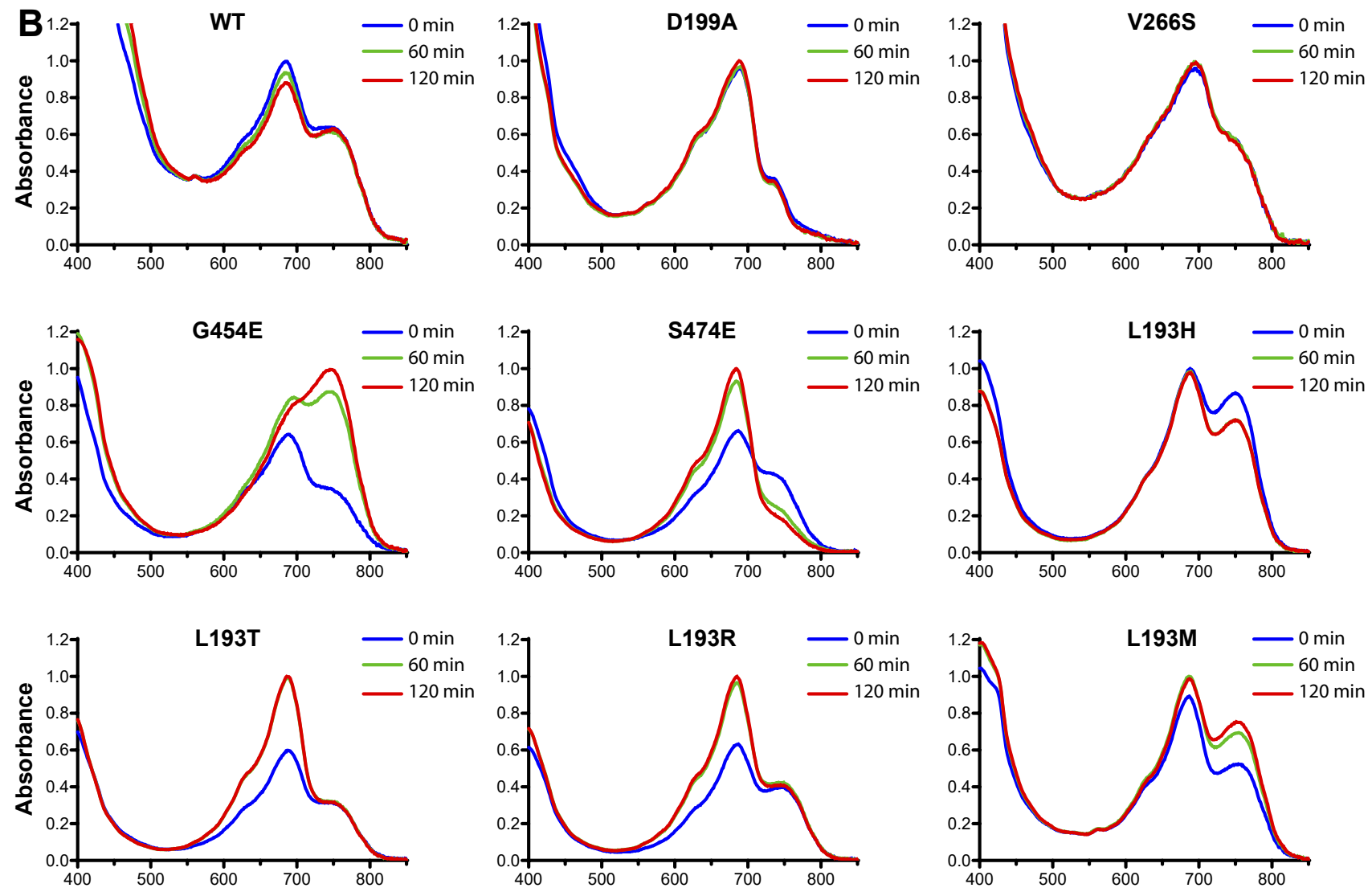

\section{.}
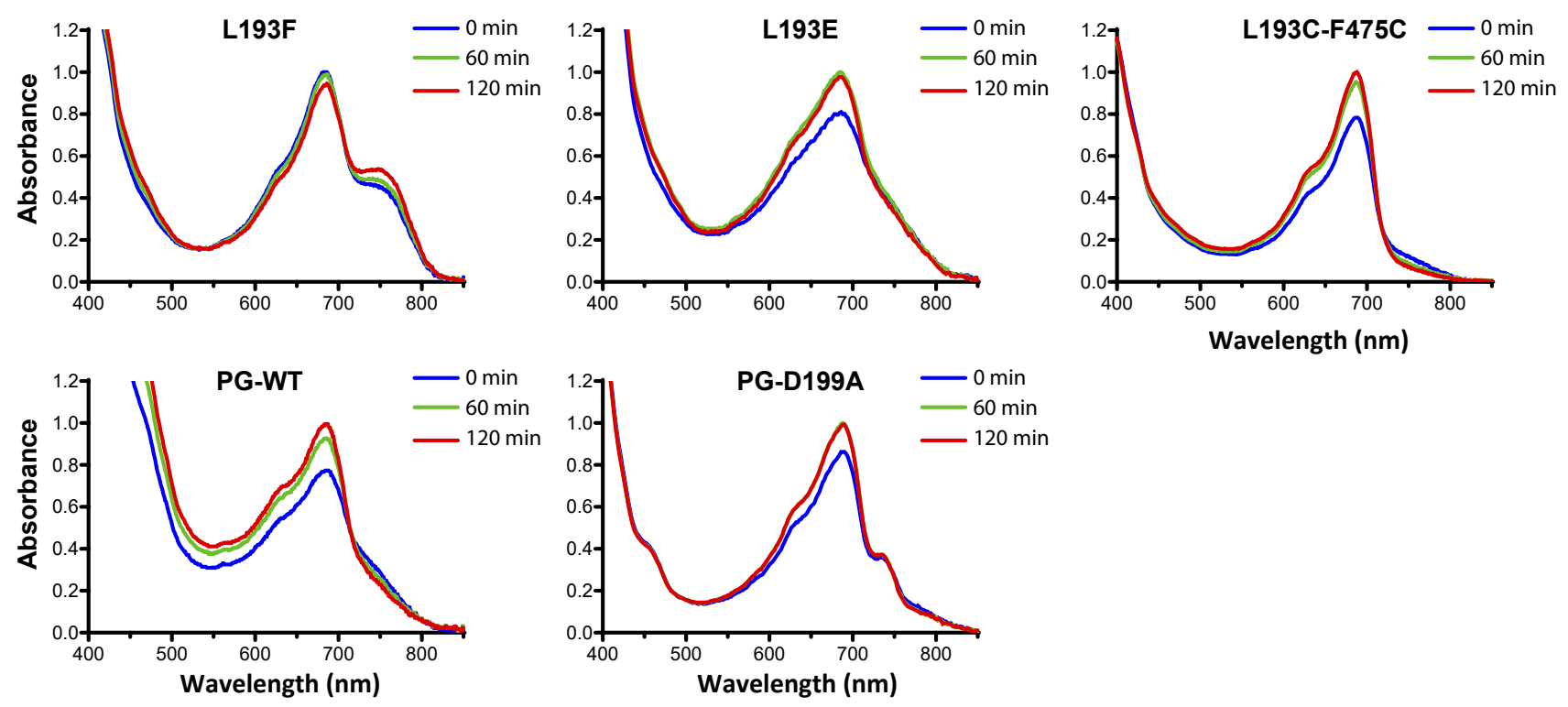